\section{PSQ-098 USING A CUSTOMISED MEDICATION VERIFICATION TOOL ON ADMISSION TO HOSPITAL IN PSYCHIATRIC PATIENTS TO REDUCE CLINICALLY RELEVANT MEDICATION DISCREPANCIES}

${ }^{1} V$ Akrum*, 'B Maat, ${ }^{2} \mathrm{~N}$ Veth. ${ }^{1}$ Elisabeth-Tweesteden Hospital, Clincal Pharmacy, Tilburg, The Netherlands; ${ }^{2}$ Elisabeth-Tweesteden Hospital, Medical Psychiatry Unit, Tilburg, The Netherlands

\subsection{6/ejhpharm-2019-eahpconf.531}

Background Medication discrepancies on admission is a common occurrence in hospitalised patients which can cause problems during hospitalisation. ${ }^{1}$ Studies have shown that medication verification on admission results in fewer discrepancies. Most medication verification studies have been carried out with a general hospital population and do not include psychiatric patients. Medication reconciliation studies in psychiatric patients are scarce. One study showed that medication verification, using a structured medication history in psychiatric patients, resulted in a more accurate overview of medication on admission. ${ }^{2}$

Purpose To reduce clinically relevant medication discrepancies in psychiatric patients admitted to the medical psychiatric unit (MPU), using a standardised or customised medication verification tool by the hospital pharmacy.

Material and methods Patients admitted to the MPU were randomised in group $\mathrm{A}, \mathrm{B}$ or $\mathrm{C}$. In group $\mathrm{A}$ medication verification was done by the physician, in group B verification was done by a pharmacy technician using a standardised tool and in group $C$ the pharmacy technician used a medication verification tool which was customised for the MPU. Medication discrepancies were assessed by comparing clinically prescribed medication to the outpatient medication records. All medication discrepancies were reviewed by a panel (two clinical pharmacists and a psychiatrist). This panel determined the clinical relevance of the medication discrepancies using the National Coordinating Council for Medication Error Reporting and Prevention index. Categories $\mathrm{E}$ to $\mathrm{I}$ were considered clinically relevant.

Results At the time of the interim analysis, 45 out of 124 patients were included (33\% males; 67\% females).

Thirty-five patients had at least one discrepancy, the mean age was 55 years and the mean number of medications was 7.1. In total, 98 discrepancies were found. Of these $27(28 \%)$ were determined clinically relevant.

Conclusion The interim analysis shows relatively more clinically relevant discrepancies in group C. This may indicate that using a customised medication verification tool could lead to fewer clinically relevant medication discrepancies and, therefore, an improvement in clinical care. Statistical testing will be used after inclusion to determine whether these initial findings can be confirmed.

\section{REFERENCES AND/OR ACKNOWLEDGEMENTS}

1. https://www.ncbi.nlm.nih.gov/pubmed/15738372

2. https://www.ncbi.n/m.nih.gov/pubmed/23959814

No conflict of interest.

\section{PSQ-099 IMPACT OF ROBOTICS ON PATIENT SAFETY AND PRODUCTIVITY}

${ }^{1} \mathrm{M}$ Al Nemari*, ${ }^{2} \mathrm{E}$ Zayed, ${ }^{2} \mathrm{~A}$ Bin Dous, ${ }^{2} \mathrm{~A}$ Malhani, ${ }^{2} \mathrm{~N}$ Al Banyan, ${ }^{2} \mathrm{~A}$ Al Rassan, ${ }^{3} \mathrm{M}$ Faqehi, ${ }^{4} \mathrm{P}$ Sharma, ${ }^{5} \mathrm{Am}$ Al Faadhel, ${ }^{5} \mathrm{~A}$ Al Sarheed, ${ }^{5} \mathrm{M}$ Gashi. ${ }^{1}$ King Fahad Medical City, Supervisor- Pharmacy Informatics and Automation, Riyadh, Saudi Arabia; ${ }^{2}$ King Fahad Medical City, Pharmacist, Riyadh, Saudi Arabia; ${ }^{3}$ King Fahad Medical City, Assistant Pharmacist, Riyadh, Saudi Arabia; ${ }^{4}$ Six Sigma, Master Black Belt, Riyadh, Saudi Arabia; ${ }^{5}$ King Fahad Medical City, Riyadh, Saudi Arabia

\subsection{6/ejhpharm-2019-eahpconf.532}

Background Automated dispensing machines are centralised medication distribution systems that provide computer-controlled storage, dispensing and tracking of medications. It is recommended as a potential mechanism to improve efficiency and patient safety. It has been proven that automation enhances the efficiency of medication distribution and its capability to reduce medication errors, increase patient safety, streamline hospital pharmacy operations and increase accuracy.

Purpose To find out the effect of automated dispensing machines versus normal workflow on turnaround time (TAT), medication error (MER) reduction and workload on staff involved in inpatient unit dose.

Material and methods Six sigma approaches were used to study the medication process before and after automation. Implementation of automated dispensing machines at the main hospital was in December 2013, medications (total 95136 units processed) were received through automation, TAT, MER and overtime of staff concerned were measured during 2014 and 2015. Same parameters were considered retrospectively for the period of January-November 2013, where medications (total 62502 units processed) were packaged, prepared and labelled manually. Data were collected after implementing automation and compared to the preavailable data.

Results By comparing manual and automated medication packaging, storage, dispensing and labelling, we found a vast reduction in TAT from $56 \mathrm{~min}$ to $17 \mathrm{~min}$ which equals $69 \%$. In addition, a dramatic decrease in preparation time was obtained, where preparing 10 items by automation was instant and did not take more than $1 \mathrm{~min}$, while the manual preparation time was $17 \mathrm{~min}$. Overtime reduction was valuable as well, where the pharmacy was able to achieve a $66 \%$ decrease in overtime (from 889 to 302 hours). MER was also reduced by $100 \%$, which had a positive impact on safety as a result of the accurate dispensing process.

Conclusion The implementation of automated dispensing machines and centralised medication distribution systems has a significantly positive impact on patient safety due to a reduction in medication errors, hence patient safety. The use of automation in healthcare has the potential to increase the quality of patient care in the hospital setting. Automation not only confirms the five rights of medication for each patient, but also allows healthcare professionals to perform tasks that improve patient care in other ways.

\section{REFERENCES AND/OR ACKNOWLEDGEMENTS}

No conflict of interest. 